\title{
ON EXISTENCE OF COMPACT OPEN NORMAL SUBGROUPS OF 0-DIMENSIONAL GROUPS
}

\author{
D. H. LEE AND T. S. WU 1
}

\begin{abstract}
ABSTRACr. Let $G$ be a locally compact 0 -dimensional group, and let $B(G)$ denote the set of all elements of $G$ whose conjugacy classes are relatively compact. We proved that the group $G$ has an open compact normal subgroup if and only if $B(G)$ is open in $G$.
\end{abstract}

Though a great deal has been learned about the structure of connected locally compact groups, the structural results on nonconnected groups seem to be very scarce. One of the reasons for this drawback seems to be due to the lack of compact open normal subgroups in general 0-dimensional locally compact groups. In this work, we characterize such groups in terms of orbits under the action of the group of inner automorphisms. An interesting corollary of this characterization is given which provides the topological version of a similar theorem due to B. H. Neumann [2].

1. Lemma. Let $G$ be a 0 -dimensional locally compact group. Then $G$ contains a compact open normal subgroup if and only if $G$ contains a compact invariant subset with nonvoid interior.

Proof. Suppose that $A$ is an invariant compact open subset of $G$ with nonvoid interior. Then $A A^{-1}$ is an invariant compact neighborhood of the identity. By a theorem of Iwasawa [1], there exists a compact normal subgroup $N$ such that $G / N$ possesses small compact open invariant neighborhoods. Since $G / N$ is also 0-dimensional, it contains small compact open normal subgroups. Thus the inverse image of any compact open normal subgroup of $G / N$ under the canonical homomorphism is then a compact open normal subgroup of $G$.

The other implication is a triviality.

2. Lemma. Let $G$ be a 0-dimensional locally compact group which does not possess any compact open normal subgroup. Then, for every compact

Received by the editors May 2, 1969.

AMS 1969 subject classifications. Primary 2210, 2220.

Key words and phrases. Locally compact groups, 0-dimensional groups, torsion elements.

1 The second author was partially supported by NSF GP-7527 and NSF g-GP8961. 
open subgroup $K$ of $G$, there exists $k \in K$ such that the set $\left\{g k g^{-1} \mid g \in G\right\}$ is not relatively compact.

Proof. Let $K$ be any compact open subgroup. Since $K$ is not normal, there are elements $k_{1} \in K$ and $a_{1} \in G$ such that $a_{1} k_{1} a_{1}^{-1} \notin K$. Define a decreasing sequence of compact open subgroups $K_{i}, i$ $=1,2, \cdots$, with $K_{1}=K$, and also sequences $\left\{a_{i}\right\},\left\{k_{i}\right\}$ with the following properties:

(i) $a_{i} \in G$ for all $i$ and $k_{i} \in K_{i} \backslash K_{i+1}, i=1,2, \cdots$,

(ii) $a_{i} K_{i+1} a_{i}^{-1} \subseteq K_{i}, i=1,2, \cdots$,

(iii) $a_{i} k_{1} k_{2} \cdots k_{i} a_{i}^{-1} \notin R_{i-1}, i=1,2, \cdots$, where $R_{i}=K_{1} \cup a_{1} k_{1} a_{1}^{-1} K_{1} \cup \cdots \cup a_{i} k_{1} k_{2} \cdots k_{i} a_{i}^{-1} K_{1}$, with $R_{0}=K_{1}$.

To see these sequences exist, suppose that $K_{1}, K_{2}, \cdots, K_{n}$; $a_{1}, a_{2}, \cdots, a_{n}$ and $k_{1}, k_{2}, \cdots, k_{n}$ have been found subject to (i), (ii) and (iii). There exists a compact open subgroup $K_{n+1}$ such that $k_{n} \notin K_{n+1}$ and $\left(a_{n} K_{n+1} a_{n}^{-1}\right) \cup K_{n+1} \subset K_{n}$. To see this, let $\bigvee$ be an open neighborhood of 1 contained in $K_{n}-\left\{k_{n}\right\}$. Since the inner automorphism induced by the element $a_{n}$ is continuous, we may assume that $a_{n} \bigvee a_{n}^{-1} \subseteq K_{n}$. Then if $K_{n+1}$ be any open compact subgroup contained in $\vee$, we have $\left(a_{n} K_{n+1} a_{n}^{-1}\right) \cup K_{n+1} \subseteq K_{n}$ with $k_{n} \notin K_{n+1}$. Suppose $g k_{1} k_{2} \cdots k_{n} k_{n+1} g^{-1} \in R_{n}$ for all $g \in G$ and all $k_{n+1} \in K_{n+1}$. Then $k_{1} \cdots k_{n} K_{n+1}$ is contained in $\bigcap_{g \in G} g^{-1} R_{n} g$. Hence $\bigcap_{g \in G} g^{-1} R_{n} g$ is a compact invariant subset of $G$ with nonvoid interior, which contradicts the assumption in view of 1 . Hence, there exist $a_{n+1} \in G$ and $k_{n+1} \in K_{n+1}$ such that $a_{n+1} k_{1} \cdots k_{n+1} a_{n+1}^{-1} \notin R_{n}$.

Now let $p_{n}=k_{1} k_{2} \cdots k_{n}, n=1,2,3, \cdots$. Since $p_{n} \in K_{1}$, for all $n=1,2, \cdots \cdot$, and since $K_{1}$ is compact, we may assume, without loss of generality, the sequence $\left\{p_{n}\right\}$ converges to an element $k \in K_{1}$. We now want to show that the set $\left\{g k g^{-1} \mid g \in G\right\}$ is not relatively compact, by showing that the sequence $\left\{a_{i} k a_{i}^{-1} \mid i=1,2, \cdots\right\}$ does not contain any convergent subsequence. Since $k=\lim p_{n}$,

$$
\begin{aligned}
a_{n} k a_{n}^{-1} & =\lim _{l} a_{n}\left(k_{1} \cdots k_{l}\right) a_{n}^{-1} \\
& =\lim _{l}\left(a_{n} k_{1} \cdots k_{n} a_{n}^{-1}\right)\left(a_{n} k_{n+1} \cdots k_{l} a_{n}^{-1}\right) \in a_{n} k_{1} \cdots k_{n} a_{n}^{-1} K_{1},
\end{aligned}
$$

because $a_{n} k_{n+1} \cdots k_{l} a_{n}^{-1}=\left(a_{n} k_{n+1} a_{n}^{-1}\right) \cdots\left(a_{n} k_{l} a_{n}^{-1}\right) \in K$. On the other hand, $a_{n} k_{1} \cdots k_{n} a_{n}^{-1} \notin R_{n-1}$. Thus $a_{i} k a_{i}^{-1} K_{1} \cap a_{j} k a_{j}^{-1} K_{1}=\varnothing$ for $i \neq j$. Since each coset $g K_{1}$ is open and closed, we see that $\left\{a_{i} k a_{i}^{-1} \mid i=1,2, \cdots\right\}$ does not converge. The same argument shows that $\left\{a_{i} k a_{i}^{-1} \mid i=1,2, \cdots\right\}$ does not contain any convergent subsequence. Hence, $\left\{g k g^{-1} \mid g \in G\right\}$ is not relatively compact. 
3. Definition. Let $G$ be a locally compact group and, for each $x \in G$, let $\Gamma(x)=\left\{g x g^{-1} \mid g \in G\right\}$. Let $B(G)$ denote the set of $x \in G$ with $\Gamma(x)$ relatively compact. Since $\Gamma(x y) \subset \Gamma(x) \Gamma(y)$, it follows that $B(G)$ is a characteristic subgroup of $G$.

Now we are ready to present the main theorem of this paper:

4. TheOREM. Let $G$ be a 0 -dimensional locally compact group. Then the following are equivalent:

(i) $G$ possesses a compact open normal subgroup.

(ii) $B(G)$ is an open characteristic subgroup of $G$.

Proof. (i) implies (ii): Let $K$ be a compact open normal subgroup of $G$. Then $K \subset B(G)$. Hence, $B(G)$ is open.

(ii) implies (i): Suppose now that $B(G)$ is open in $G$. If there is no compact open normal subgroup of $G$, then, for any compact open subgroup $K$ which is contained in $B(G)$, by Lemma 2 , there exists a $k \in K$ such that $\Gamma(k)$ is not relatively compact. Hence, $k \notin B(G)$ which is an obvious contradiction. Hence, $G$ must contain a compact open normal subgroup.

5. Definition. Let $G$ be a locally compact group. An element $g \in G$ is said to be compact, if $g$ is contained in a compact subgroup of $G$.

6. Corollary. Let $G$ be a locally compact 0-dimensional group such that $B(G)=G$. Then the set $p(G)$ of all compact elements of $G$ forms an open characteristic subgroup of $G$.

Proof. Since $B(G)=G, G$ contains a compact open normal subgroup $K$ by Theorem 4. That $B(G)=G$ also implies that $B(G / K)$ $=G / K$. Thus the conjugate class of every element in the discrete group $G / K$ is finite. By a theorem of Neumann [2], the set $p(G / K)$ of all torsion elements forms a subgroup. Let $\pi: G \rightarrow G / K$ be the quotient morphism. Then it is easy to see that $p(G)=\pi^{-1}(p(G / K))$. Hence $p(G)$ is a subgroup of $G$. Since there are small compact open subgroups in $G, p(G)$ is open.

\section{BIBLIOGRAPHY}

1. K. Iwasawa, Topological groups with invariant compact neighborhoods of the identity, Ann. of Math. (2) 54 (1951), 345-348. MR 13, 206.

2. B. H. Neumann, Groups with finite classes of conjugate elements, Proc. London Math. Soc. (3) 1 (1951), 178-187. MR 13, 316.

Case Western Reserve University, Cleveland, Ohio 44106 